\title{
Moving Synergistically Acting Drug Combinations to the Clinic by Comparing Sequential versus Simultaneous Drug Administrations $\$$
}

\author{
Saketh S. Dinavahi, Mohammad A. Noory, Raghavendra Gowda, Joseph J. Drabick, \\ Arthur Berg, Rogerio I. Neves, and Gavin P. Robertson \\ Division of Hematology-Oncology (J.J.D.); Departments of Pharmacology (S.S.D., M.A.N., R.G., R.I.N., G.P.R.), Medicine (J.J.D.), \\ Public Health Sciences (A.B.), Dermatology (R.I.N., G.P.R.), Surgery (R.I.N., G.P.R.), and Pathology (G.P.R.); Melanoma and Skin \\ Cancer Center (S.S.D., M.A.N., R.G., J.J.D., A.B., R.I.N., G.P.R.); Foreman Foundation for Melanoma Research (R.G., G.P.R.); \\ and the Melanoma Therapeutics Program (R.G., R.I.N., G.P.R.), Pennsylvania State University College of Medicine, Hershey, \\ Pennsylvania
}

Received September 28, 2017; accepted December 12, 2017

\section{ABSTRACT}

Drug combinations acting synergistically to kill cancer cells have become increasingly important in melanoma as an approach to manage the recurrent resistant disease. Protein kinase $B(A K T)$ is a major target in this disease but its inhibitors are not effective clinically, which is a major concern. Targeting AKT in combination with WEE1 (mitotic inhibitor kinase) seems to have potential to make AKT-based therapeutics effective clinically. Since agents targeting AKT and WEE1 have been tested individually in the clinic, the quickest way to move the drug combination to patients would be to combine these agents sequentially, enabling the use of existing phase I clinical trial toxicity data. Therefore, a rapid preclinical approach is needed to evaluate whether simultaneous or sequential drug treatment has maximal therapeutic efficacy, which is based on a mechanistic rationale. To develop this approach, melanoma cell lines were treated with AKT inhibitor AZD5363 [4-amino-
$N$-[(1S)-1-(4-chlorophenyl)-3-hydroxypropyl]-1-(7H-pyrrolo[2,3-d]pyrimidin-4-yl)piperidine-4-carboxamide] and WEE1 inhibitor AZD1775 [2-allyl-1-(6-(2-hydroxypropan-2-yl)pyridin-2-yl)-6-((4(4-methylpiperazin-1-yl)phenyl)amino)-1H-pyrazolo[3,4- $d$ ]pyrimidin$3(2 H)$-one] using simultaneous and sequential dosing schedules. Simultaneous treatment synergistically reduced melanoma cell survival and tumor growth. In contrast, sequential treatment was antagonistic and had a minimal tumor inhibitory effect compared with individual agents. Mechanistically, simultaneous targeting of AKT and WEE1 enhanced deregulation of the cell cycle and DNA damage repair pathways by modulating transcription factors p53 and forkhead box M1, which was not observed with sequential treatment. Thus, this study identifies a rapid approach to assess the drug combinations with a mechanistic basis for selection, which suggests that combining AKT and WEE1 inhibitors is needed for maximal efficacy.

\section{Introduction}

Despite recent advances in targeted therapies and immune system modulators, development of resistance continues to be a major concern for melanoma treatment, requiring the

This work was supported by the National Institutes of Health National Cancer Institute [Grants R01-CA136667, R01-CA1138634, and R01CA127892], the Foreman Foundation for Melanoma, the Geltrude Foundation, and the Penn State Chocolate Tour Cancer Research Fund.

https://doi.org/10.1124/mol.117.110759.

S This article has supplemental material available at molpharm. aspetjournals.org. identification of drug combinations to retard this process (Villanueva et al., 2010; Paraiso et al., 2011). Drug combinations effective for treating melanoma often involve the use of agents that have undergone U.S. Food and Drug Administration (FDA)-required toxicity evaluation as single agents in human trials (Al-Lazikani et al., 2012). Movement of drug combinations rapidly to the clinic is not generally a fast process; however, if the agents can be combined sequentially, this process can move more quickly and efficiently. Furthermore, a solid mechanistic basis for the selection would be needed. If the sequential approach were feasible, it could

ABBREVIATIONS: AKT, protein kinase B; AZD1775, 2-allyl-1-(6-(2-hydroxypropan-2-yl)pyridin-2-yl)-6-((4-(4-methylpiperazin-1-yl)phenyl)amino)1H-pyrazolo[3,4-d]pyrimidin-3(2H)-one; AZD5363, 4-amino- $N$-[(1S)-1-(4-chlorophenyl)-3-hydroxypropyl]-1-(7H-pyrrolo[2,3- $d]$ pyrimidin-4-yl)piperidine-4-carboxamide; Cl, combination index; DMEM, Dulbecco's modified Eagle's medium; DMSO, dimethylsulfoxide; FDA, U.S. Food and Drug Administration; GDC0068 [(2S)-(4-chlorophenyl)-1-[4-[(5R, 7R)-6,7-dihydro-7-hydroxy-5-methyl-5H-cyclopentapyrimidin-4-yl]-1-piperazinyl]3-[(1-methylethyl)amino]-1-propanone; MTS, 3-(4,5-dimethylthiazol-2-yl)-5-(3-carboxymethoxyphenyl)-2-(4-sulfophenyl)-2H-tetrazolium. 
potentially simplify the process, as the toxicity of the individual agents would be known (http://www.fda.gov/downloads/ Drugs/GuidanceComplianceRegulatoryInformation/Guidances/ UCM236669.pdf). In contrast, combining agents requires additional toxicity assessments and the filing of a new Investigational New Drug application with the FDA, which adds cost and increases time to clinical evaluation (http://www.fda.gov/ downloads/Drugs/GuidanceComplianceRegulatoryInformation/ Guidances/UCM236669.pdf).

Protein kinase B (AKT) signaling is activated in up to $70 \%$ of sporadic melanomas, and targeting this protein in preclinical models effectively inhibits tumor development (Stahl et al., 2004; Cheung et al., 2008; Davies et al., 2008; Shao and Aplin, 2010). Unfortunately, these studies did not translate clinically and AKT inhibitors tested in patients with melanoma had little efficacy (Pal et al., 2010; Dinavahi et al., 2015). Therefore, a screen was undertaken to identify targets and agents that could be combined with AKT inhibition to more effectively target this pathway (Pal et al., 2010). Genetic and pharmacologic targeting of AKT and WEE1 was found to be an effective approach, leading to dramatic and highly synergistic killing of melanoma cells in culture and in mice tumors (Kuzu et al., 2017a,b).

WEE1 kinase lies downstream of V600E-BRAF in the mitogen-activated protein kinase signaling cascade (Sharma et al., 2013). Interestingly, combined targeting of AKT and V600E-BRAF was additive, while targeting AKT and downstream WEE1 was highly synergistic, making this a potentially more important actionable target (Kuzu et al., 2017a,b). WEE1 responds to cellular DNA damage by regulating the cell cycle through phosphorylation-mediated inactivation of cyclin-dependent kinase 1 , which halts the cell cycle in the G2/M phase until the damage is repaired (Watanabe et al., 1995; Madhunapantula et al., 2013).

For this study, existing drugs targeting AKT and WEE1 that have undergone phase I evaluation were tested (Pal et al., 2010). Simultaneous treatment with WEE1 inhibitor AZD1775 [2-allyl-1-(6-(2-hydroxypropan-2-yl)pyridin-2-yl)-6((4-(4-methylpiperazin-1-yl)phenyl)amino)- $1 H$-pyrazolo[3,4- $d]$ pyrimidin-3(2H)-one] and with AKT inhibitors AZD5363 [4-amino- $N$-[(1S)-1-(4-chlorophenyl)-3-hydroxypropyl]-1-(7Hpyrrolo[2,3- $d]$ pyrimidin-4-yl)piperidine-4-carboxamide] or GDC0068 [(2S)-(4-chlorophenyl)-1-[4-[(5R,7R)-6,7-dihydro-7hydroxy-5-methyl-5H-cyclopentapyrimidin-4-yl]-1-piperazinyl] -3-[(1-methylethyl)amino]-1-propanone] synergistically killed cultured melanoma cells and inhibited melanoma tumor growth by $>90 \%$, irrespective of BRAF mutational status (Kuzu et al., 2017a). Mechanistically, simultaneous drug treatment enhanced the effects modulated by each of the individual drugs, leading to significant deregulation of the cell cycle and DNA damage repair pathways mediated through transcription factors p53 and FOXM1 (Kuzu et al., 2017a,b).

To design a strategy for clinical evaluation, a rapid approach was needed to assess whether sequential drug treatment would have the same effect as simultaneous drug treatment and mechanistically validate the approach. Simultaneous treatment with the drug combination in cultured melanoma cells was highly synergistic in contrast to sequential treatment, which was antagonistic. Similarly, simultaneous treatment led to a $>90 \%$ reduction of xenograft tumor development without toxicity. In contrast, sequential treatment was less effective and showed signs suggestive of toxicity.
Simultaneous treatment increased p53 pathway activity while decreasing FOXM1 signaling, which did not occur with sequential treatment, providing a solid mechanistic basis for selecting the simultaneous rather than sequential approach for clinical evaluation of the drug combination.

\section{Materials and Methods}

Cell Lines and Culture Conditions. UACC 903, $1205 \mathrm{Lu}$, SK-MEL-28, and WM164 melanoma cell lines were grown in Dulbecco's modified Eagle's medium with 1\% GlutaMAX (Life Technology, Waltham, MA) and $10 \%$ fetal bovine serum in a $37^{\circ} \mathrm{C}$ humidified $5 \%$ $\mathrm{CO}_{2}$ incubator. Cell lines were periodically monitored for genotypic characteristics, phenotypic behavior, and tumorigenic potential to confirm identity (Gowda et al., 2017).

Drug Compounds. AstraZeneca (Wilmington, DE) generously gifted AZD5363 and AZD1775. The compounds were dissolved in $20 \mathrm{mM}$ dimethylsulfoxide (DMSO) for cell culture-based synergy analysis. For tumor-based synergy studies, drugs were prepared in $0.5 \%$ methylcellulose, $1.0 \%$ Tween 80 , and $5 \%$ DMSO and $200 \mu \mathrm{l}$ was administered orally (Kuzu et al., 2017a).

Cell Culture-Based Synergy Analysis. Cell viability was assessed by the MTS [3-(4,5-dimethylthiazol-2-yl)-5-(3-carboxymethoxyphenyl)-2-(4-sulfophenyl)-2H-tetrazolium] assay as described previously (Kuzu et al., 2017a). Cells were treated with AKT inhibitor AZD5363, WEE1 inhibitor AZD1775, and a combination of both at the same time for simultaneous treatment for 72 hours. For sequential treatment, $1 \mu \mathrm{M}$ AZD1775 was administered for 24 hours, followed by treatment with various concentrations of AZD5363 for 48 hours. Combination index (CI) values were calculated using CalcuSyn software (Biosoft, Cambridge, UK) (Kuzu et al., 2017a,b). Briefly, data were expressed as the fraction of cells affected by the dose in drug-treated cells compared with DMSO-treated cells. The CalcuSyn program is based on the Chou-Talalay method according to the following equation: $\mathrm{CI}=(\mathrm{D}) 1 /(\mathrm{D} x) 1+(\mathrm{D}) 1(\mathrm{D}) 2 /(\mathrm{D} x) 1(\mathrm{D} x) 2$, where (D) 1 and (D) 2 are the doses of drug 1 and drug 2 that have the same $x$ effect when used alone. CI values $<0.9$ were considered synergistic, values $>1.1$ were considered antagonistic, and values ranging from 0.9 to 1.1 were considered additive (Chou, 2010).

Tumor-Based Synergy Analysis. Xenografted tumor assessment studies were undertaken in athymic-Foxn $1^{\text {nu }}$ nude mice as described previously (Kuzu et al., 2017a). Eight days after cell injection when a fully vascularized tumor had formed, daily oral treatments with AZD1775 (50 mg/kg) and AZD5363 (150 mg/kg) were initiated either alone or in combination for simultaneous treatment. For sequential treatment, the oral regimen was adapted from clinical usage and included twice-daily treatment with AZD5363 $(130 \mathrm{mg} / \mathrm{kg})$ for 4 days and twice-daily treatment with AZD1775 $(30 \mathrm{mg} / \mathrm{kg})$ for 3 days. Tumor volumes and animal weights were measured on alternate days $(N=8)$. At the end of the drug treatment, blood samples were collected; the serum was separated, and major organ biomarkers were assessed.

Mechanistic Basis for Synergy Efficacy. Western blotting was used to determine the mechanistic basis for selection of the dosage regimen as described previously (Kuzu et al., 2017a). For simultaneous treatment, melanoma cells were treated with AZD1775 and AZD5363 for 48 hours. For sequential treatment, cells were treated with AZD1775 for 24 hours and AZD5363 for another 24 hours. p53, $\mathrm{H} 2 \mathrm{~A}$ histone member $\mathrm{X}$, and secondary antibodies were from Santa Cruz Biotechnology (Dallas, TX) and FOXM1 was from Cell Signaling Technology (Danvers, MA).

Statistical Analysis. Descriptive statistics including means \pm S.E. for the serum toxicity parameters are provided in Table 1 . The measurements were analyzed with a one-way analysis of variance to identify statistically significant differences among the four treatment groups (control, AZD5363, AZD1775, and combination). Because eight different parameters were considered, a Bonferroni adjustment was 


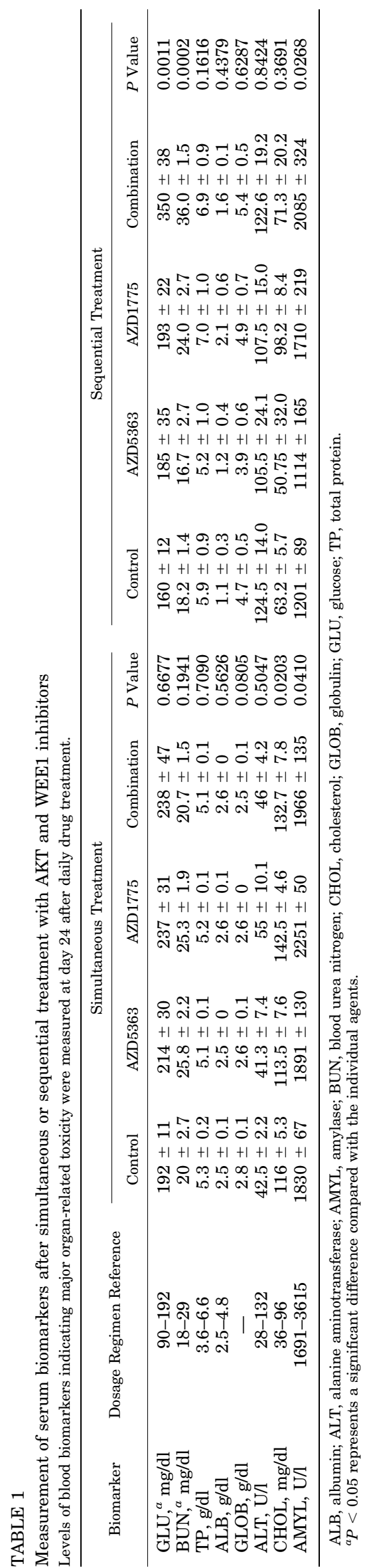

applied (by multiplying the $P$ value by 8 ) to adjust for multiple testing. The sequential and simultaneous experiments were analyzed independently. For comparison between more than two groups, one-way analysis of variance was used followed by Dunnett's test as a post hoc analysis. For analysis of xenograft data, two-way analysis of variance was used followed by Dunnett's test as a post hoc analysis. GraphPad Prism software (version 5.0; GraphPad Software Inc., La Jolla, CA) was used. Results with a $P$ value $<0.05$ (95\% confidence interval) were considered significant. The experiment-wise error threshold was $5 \%$.

\section{Results}

Assessing Whether Simultaneous versus Sequential Targeting of AKT and WEE1 More Effectively Kills Cultured Melanoma Cells. Targeting of AKT and WEE1 after simultaneous or sequential treatment was tested on cultured UACC 903 or $1205 \mathrm{Lu}$ cell lines (Fig. 1). A single treatment with1 $\mu \mathrm{M}$ AZD1775 or $0.31-20 \mu \mathrm{M}$ AZD5363 reduced cell viability by $20 \%-50 \%$ in 72 hours for UACC 903 cells (Fig. 1A, left). In contrast, a combination of the two agents led to a $80 \%$ decrease in cell viability (Fig. 1A, left). The calculated CI values ranging from 0.1 to 0.3 suggested strong synergism (Fig. 1B, left). For sequential treatment, UACC 903 cells were treated with AZD5363 for 24 hours and were then subsequently treated with AZD1775 (Fig. 1A, right). However, sequential treatment led to an antagonistic relationship between the two agents, as evident from the calculated CI values $>2.0$ (Fig. 1B, right). An alternate approach, with cells treated first with AZD1775 and then with AZD5363, was also employed, with a similar antagonistic response between the drugs (data not shown). Comparable synergistic results were obtained with the $1205 \mathrm{Lu}$ cell line, suggesting that synergy between AKT and WEE1 inhibitors is not a cell line-specific phenomenon (Fig. 1, C and D).

Evaluating Simultaneous versus Sequential Targeting of AKT and WEE1 Synergistically Inhibited Xenografted Melanoma Tumor Growth. To assess whether simultaneous or sequential regimens of AZD5363 and AZD1775 would synergistically inhibit tumor growth, efficacy of the drug combinations was evaluated in UACC 903 and $1205 \mathrm{Lu}$ xenografts (Fig. 2). For simultaneous treatment, oral administrations of AZD5363 (150 mg/kg), AZD1775 (50 $\mathrm{mg} / \mathrm{kg}$ ), or their combination were initiated 8 days after melanoma cell implantation when the tumors were vascularized and treatment continued daily. For sequential treatment, oral administrations of AZD5363 $(130 \mathrm{mg} / \mathrm{kg})$ twice daily were initiated for 4 days, followed by AZD1775 (30 mg/kg) twice daily for the next 3 days. The dosage for simultaneous treatment was established in house and used the doses calculated based on the studies with individual agents (Kuzu et al., 2017a). The dosage for sequential treatment was established in consultation with AstraZeneca to best mimic the regimen used for the phase I trials of AZD5363 (ClinicalTrials.gov identifier NCT02338622) and AZD1775 (NCT01357161). Individually, AZD1775 and AZD5363 led to a $30 \%$ and $50 \%$ reduction in tumor sizes in UACC 903 cells, respectively, whereas simultaneous treatment led to a $90 \%$ decrease (Fig. 2A). In sequential treatment, AZD5363 and AZD1775 individually led to a $50 \%$ and $15 \%$ reduction in tumor sizes, respectively (Fig. 2B). The combination led to only a $50 \%$ reduction in tumor volume, with no difference between the AZD5363-treated and combination-treated groups 
A

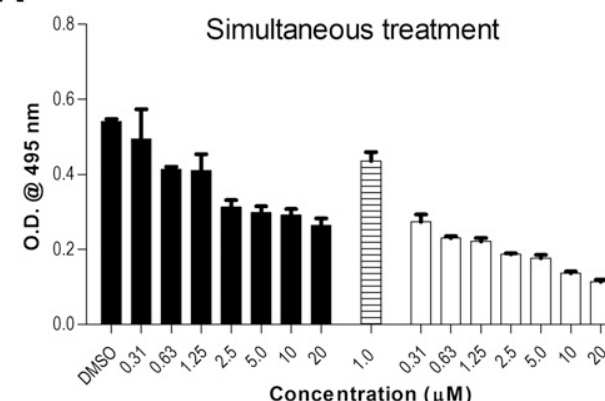

B

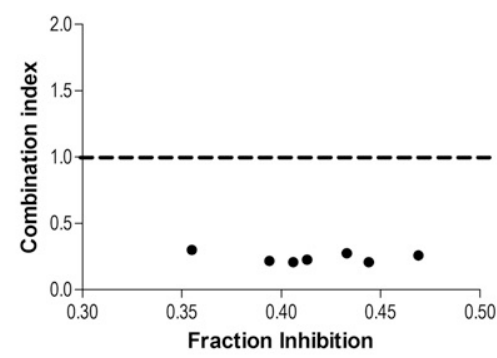

AZD5363 (0.3-20 $\mu \mathrm{M})$

C

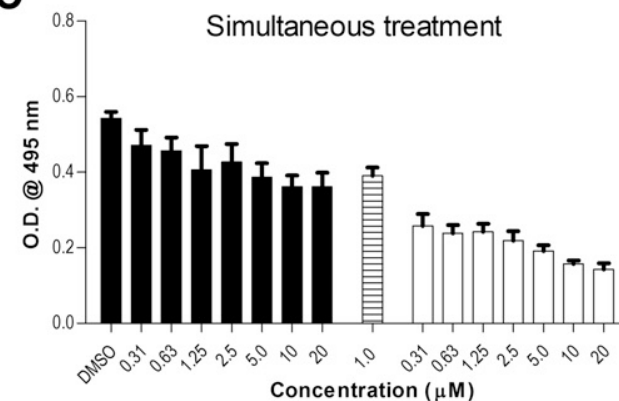

D

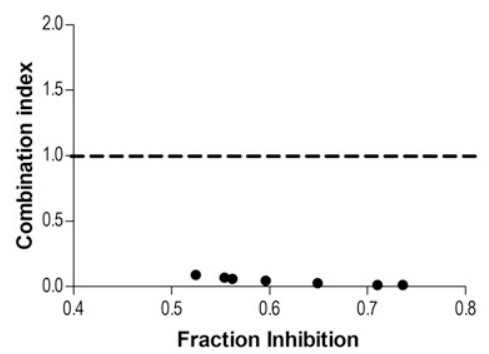

AZD5363 (0.3-20 $\mu \mathrm{M})$
UACC 903

I Sequential treatment

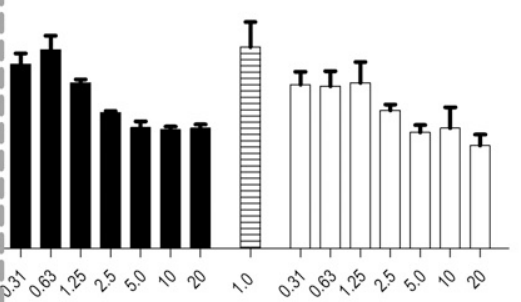

Concentration $(\mu \mathrm{M})$

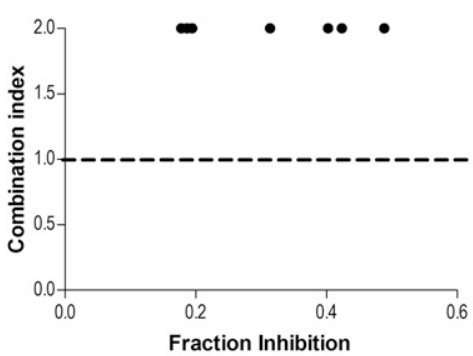

AZD5363 (0.3-20 $\mu \mathrm{M})+$ AZD1775 (1 $\mu \mathrm{M})$

1205 Lu

Sequential treatment

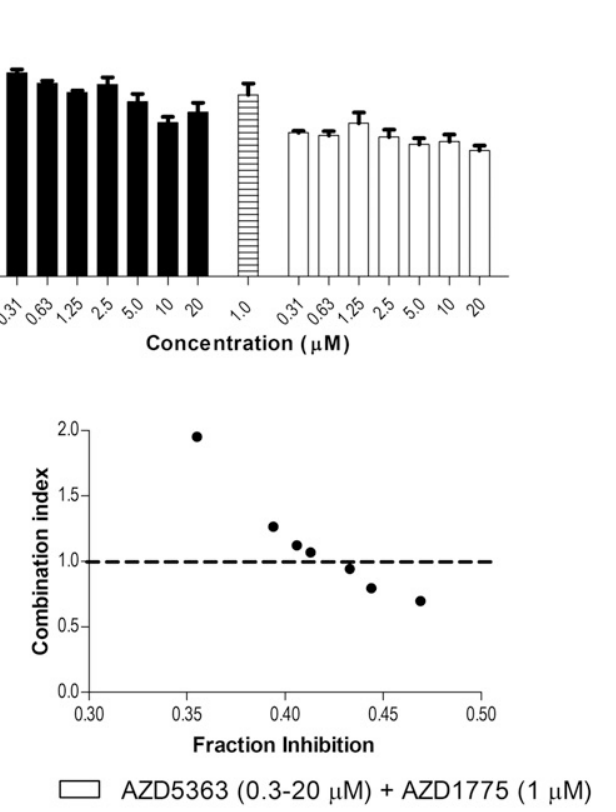

Fig. 1. Comparing simultaneous vs. sequential treatment with AKT and WEE1 inhibitors to inhibit cultured melanoma cell survival. (A and C) Dose-response curves of melanoma cell lines UACC 903 (A) or $1205 \mathrm{Lu}(\mathrm{C})$ simultaneously or sequentially treated with AZD5363 and AZD1775. (B and D) For synergy analysis, the concentrations of each drug were varied and CI values were calculated.
(Fig. 2B). For the simultaneous treatment regimen, no significant changes were observed in animal body weights (inset in Fig. 2A) or blood biomarkers indicative of vital organ function (Table 1). Although there were no changes in animal body weights for the sequential treatment regimen, significant differences were observed in blood glucose and blood urea nitrogen levels in serum, suggesting possible toxicity (Table 1). Similar results were observed in xenograft studies with $1205 \mathrm{Lu}$ cell line (Fig. 2, B and D), where simultaneous treatment led to a $90 \%$ tumor reduction compared with a $50 \%$ reduction for the sequential drug approach. Morbidity and subsequent death occurred in one of the sequential combination groups in the $1205 \mathrm{Lu}$ xenograft study (Fig. 2D, inset; death of the animal indicated by a phi symbol), further suggesting possible toxicity of the sequential dosage regimen.

Simultaneous But Not Sequential Targeting of AKT and WEE1 Enhanced the Effects of the Signaling Pathways. Simultaneously targeting AKT and WEE1 demonstrated synergism due to enhanced modulation of pathways regulated by transcription factors p53 and FOXM1 (Kuzu et al., 2017a). To validate the mechanism for the efficacy of simultaneous rather than sequential targeting, Western blotting analysis of effects of the drugs on p53 and FOXM1 protein levels was performed. For simultaneous treatment, 

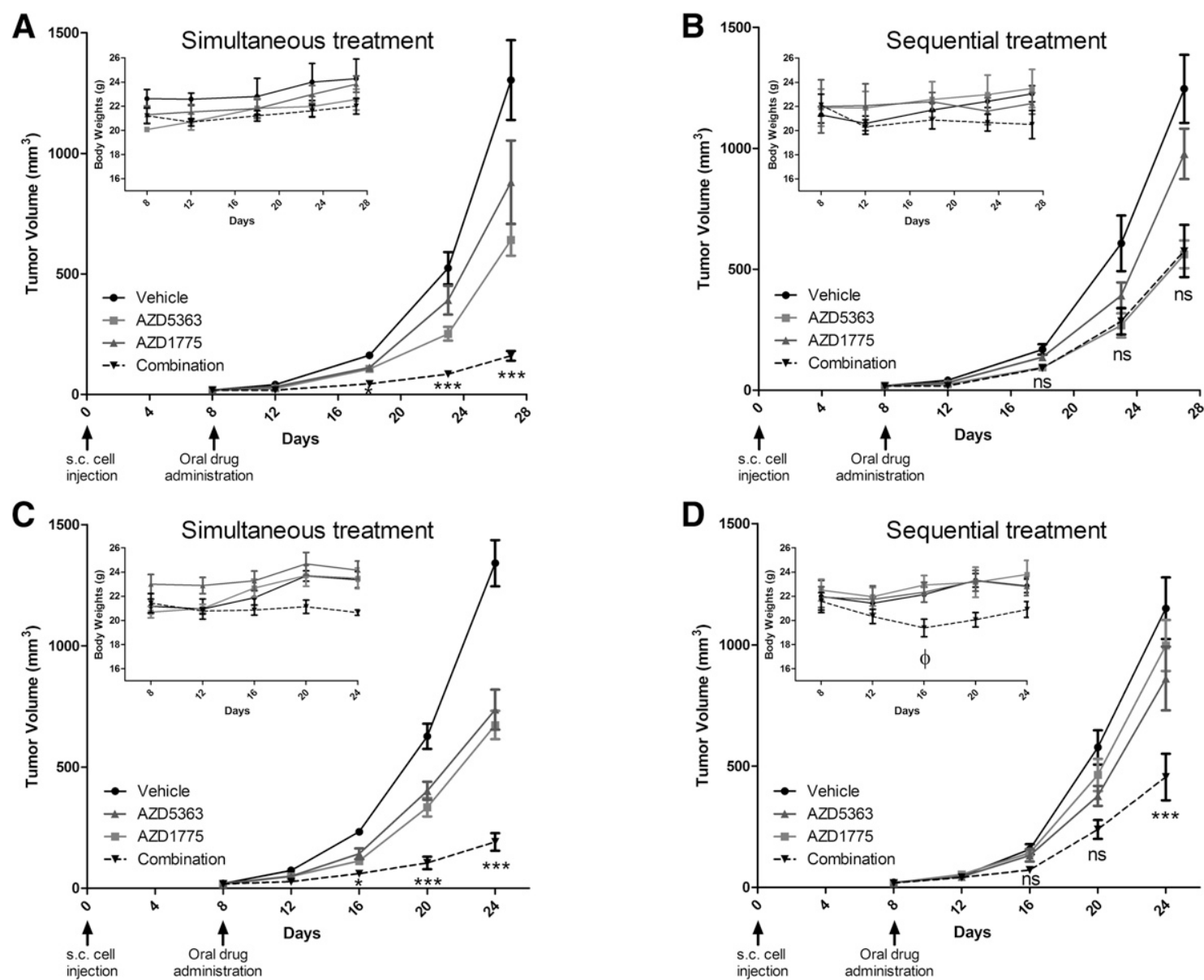

Fig. 2. Evaluation of simultaneous and sequential treatment with AKT and WEE1 targeting drugs to inhibit melanoma tumor growth. (A-D) Inhibition of UACC 903 (A and B) and $1205 \mathrm{Lu}(\mathrm{C}$ and D) xenografts after simultaneous (A and C) or sequential (B and D) treatments with AZD5363 and/or AZD1775 $(N=8)$. The insets depict animal weights to indicate gross animal toxicity of the dosage regimen. The phi symbol indicates animal mortality within the experiment. $* P<0.05 ; * * * P<0.001$.

melanoma cells were treated with AZD5363 alone or in combination with AZD1775 for 48 hours. Similarly, for sequential treatment, cells were treated with AZD5363 for 24 hours followed by a washout with phosphate-buffered saline and then treated with AZD1775 for another 24 hours. Simultaneous treatment led to a 17.5-fold and 7.5-fold increase in pH2AX and p53, respectively (Fig. 3). In stark contrast, sequential treatment led to only a 1.3 -fold and 2.7 -fold increase in pH2AX and p53, respectively. Similar results were observed for $1205 \mathrm{Lu}$ cells. To substantiate the significance of p53 activity in the mechanism for synergy, cell viability experiments were performed in TP53 mutated (dominant-negative) melanoma cell lines SK-MEL-28 and WM164 (Supplemental Fig. 1). No synergy was observed following the simultaneous treatment with AZD1775 and AZD5363, indicating that p53 activity is essential for synergy between AKT and WEE1 inhibitors. Collectively, the data suggested that the synergy between AZD5363 and AZD1775 required an increase in $\mathrm{pH} 2 \mathrm{AX}$ and p53 levels in cells, which is not enhanced in sequential treatment compared with simultaneous treatment (Fig. 3B).

\section{Discussion}

The underlying rationale for drug combination treatment in cancer has been to coadminister drugs that act by different mechanisms, thereby increasing tumor cell killing while reducing the likelihood of drug resistance (Al-Lazikani et al., 2012). To rapidly move drug combinations to the clinic, studies need to compare the effects of simultaneous versus sequential administration of targeted therapy and select the best approach based on a solid mechanistic justification (Wang et al., 2012, 2013; Fung et al., 2013; Wild et al., 2013; Dos Santos et al., 2015; Song et al., 2015). If sequential administration is as effective as simultaneous administration, existing clinical trial toxicity data can be used to expedite clinical testing.

Although many AKT inhibitors are effective in preclinical studies, they fail in clinical trials due to lack of efficacy (Davies et al., 2012). AZD5363 is a promising ATP-competitive type I inhibitor of AKT signaling with a potency of $<10 \mathrm{nM}$ for all three AKT isoforms (Davies et al., 2012). Likewise, WEE1 is expressed at high levels in many cancers, including melanoma, where this protein regulates cell cycle progression by controlling the G2/M checkpoint through catalyzing the inhibitory phosphorylation of cyclin-dependent kinase 1 (Madhunapantula et al., 2013; Visconti et al., 2016). As a single agent, AZD1775 reduced tumor development in several preclinical cancer models (Guertin et al., 2013; Do et al., 2015). Furthermore, a recent phase I trial led to a partial response in 2 of 25 enrolled patients (Guertin et al., 2013; Do et al., 2015). Currently, AZD1775 is being tested in 20 clinical trials in 
A

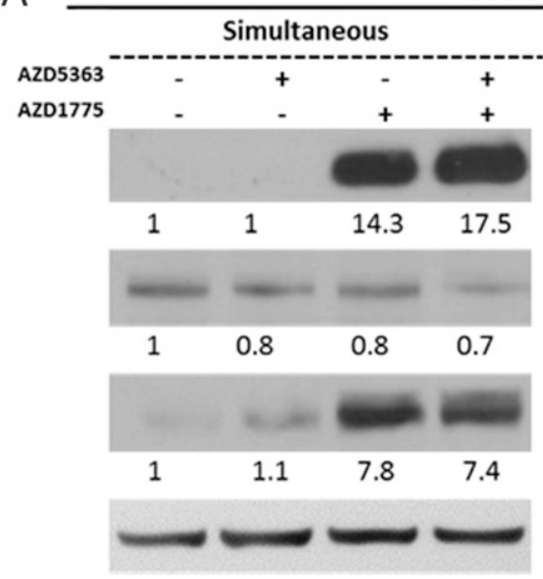

UACC 903
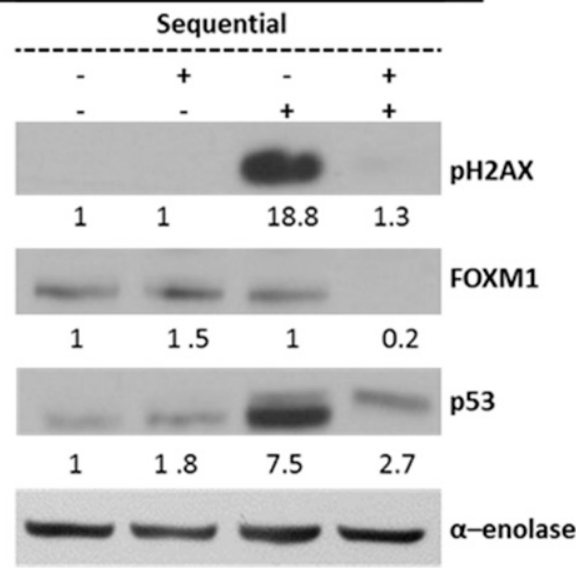

$1205 \mathrm{Lu}$
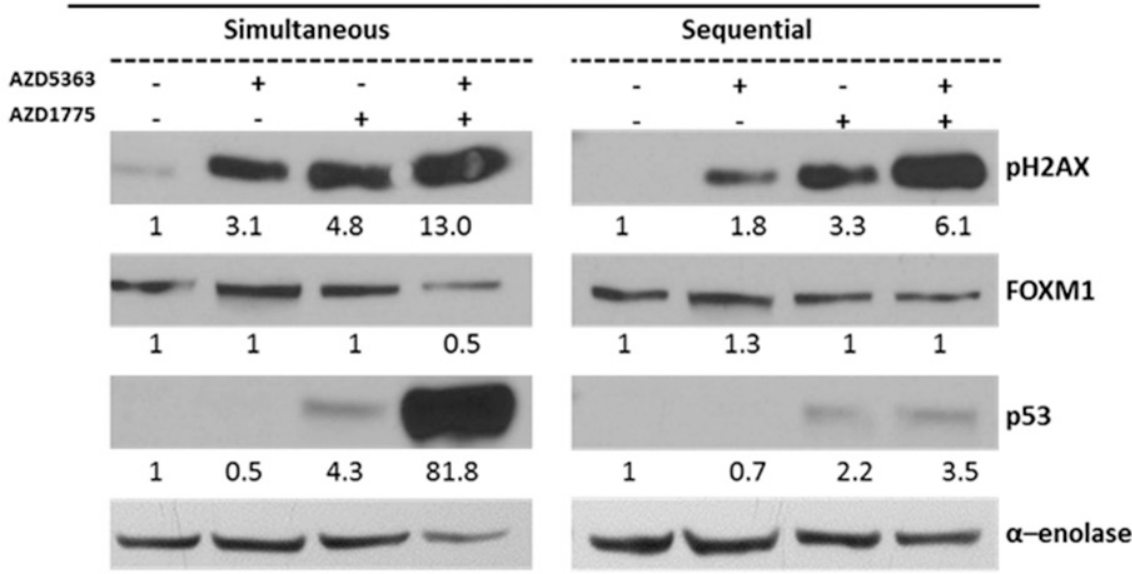

B

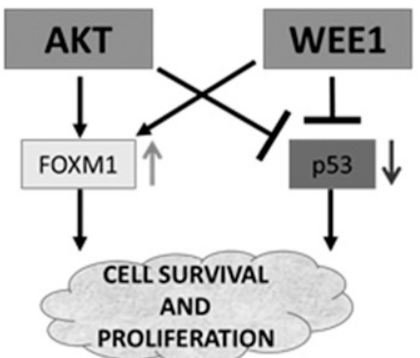

AZD5363

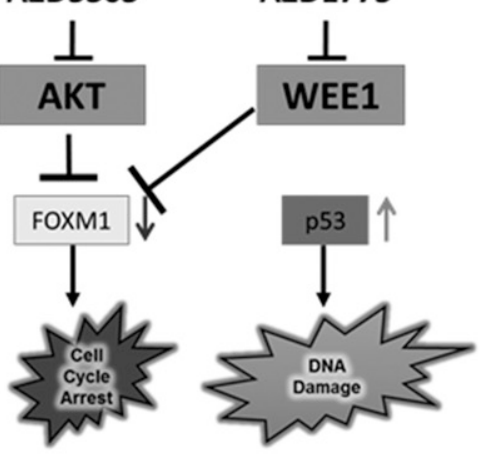

Fig. 3. Effects of simultaneous or sequential treatment with AKT or WEE1 inhibitors on p53 and FOXM1 pathway signaling. (A) Simultaneous but not sequential targeting of AKT and WEE1 enhanced the inhibitory effects occurring when targeting each pathway individually. $\alpha$-Enolase served as a control for protein loading. Numbers in (A) represent quantification of protein normalized to loading control using ImageJ software (National Institutes of Health, Bethesda, MD). (B) Combination of WEE1 and AKT inhibitors caused increased p53 and decreased FOXM1 (signaling cascade). various cancer types, including solid tumors, where it is combined with DNA-damaging agents such as carboplatin, cisplatin, paclitaxel, temozolomide, or UV irradiation (Visconti et al., 2016).

Current clinical testing of AZD5363 uses $240-480 \mathrm{mg}$ twice daily for a 4-day-on and 3-day-off cycle (NCT02338622, NCT02121639, NCT0220837, and NCT01226316). Similarly, clinical testing for AZD1775 includes $225 \mathrm{mg}$ twice daily for a 3-day-on and 4-day-off cycle (NCT01357161 and NCT01164995). The dosage regimen for sequential treatment used for this study was established to mimic the current individual trials of both AZD5363 and AZD1775. Hence, a 4-day-on and 3-day-off AZD5363 cycle with intermittent usage of AZD1775 was employed for sequential treatment. The dosage regimen of AZD1775 and AZD5363 for simultaneous treatment was determined based on previous empirical preclinical results with individual agents; synergy analysis inhibiting cultured melanoma cell lines and the 1:3 ratio of AZD1775 to AZD5363 for inhibiting melanoma xenografts (Kuzu et al., 2017a).

Simultaneous targeting of AZD5363 and AZD1775 inhibited melanoma tumor growth more synergistically than sequential 
treatment and did not lead to obvious toxicity. In contrast, sequential treatment elevated blood glucose and urea nitrogen levels. A possible explanation for reduced toxicity when using the simultaneous approach could be due to the lower amount of each drug used per day (150 mg/kg every other day and $50 \mathrm{mg} / \mathrm{kg}$ every other day) compared with sequential treatment ( $130 \mathrm{mg} / \mathrm{kg}$ twice daily and $30 \mathrm{mg} / \mathrm{kg}$ twice daily for AZD5363 and AZD1775, respectively).

The mechanistic basis for synergism was validated with only simultaneous treatment and did not occur with sequential treatment. In melanoma cells, p53 activity is tightly regulated by both AKT and WEE1 pathways (Kuzu et al., 2017a). WEE1 inhibition induces DNA damage, as indicated by the upregulation of phospho-H2AX and p53 signaling (Kuzu et al., 2017a). AKT interferes with the DNA damage response by inducing ubiquitinmediated degradation of p53 and stimulating the catalytic activity of Polo like kinase, which inhibits the proapoptotic functions of p53 (Kuzu et al., 2017a). Simultaneous drug treatment enhances p53 activity due to the increase in both pathways, whereas sequential treatment causes an increase in p53 only due to one dominant pathway and thereby limits this activity (Kuzu et al., 2017a).

This study stratifies a rapid approach to evaluate whether simultaneous versus sequential drug combinations are effective and could advance drug combinations rapidly to the clinic. This work provides novel insights regarding the dosage regimen for AKT and WEE1 drug combinations and a solid mechanistic rationale for selecting a simultaneous, rather than sequential, treatment strategy. Furthermore, this approach demonstrates the importance of increasing p53 and H2AX levels for enhanced efficacy of AKT and WEE1 inhibitors, suggesting the potential biomarkers for evaluating the drug combination in clinical trials.

\section{Authorship Contributions}

Participated in research design: Dinavahi, Noory, Gowda, Drabick, Berg, Neves, Robertson.

Conducted experiments: Dinavahi, Noory, Gowda, Neves, Robertson.

Contributed new reagents or analytic tools: Drabick, Robertson.

Performed data analysis: Dinavahi, Noory, Gowda, Drabick, Berg, Neves, Robertson.

Wrote or contributed to the writing of the manuscript: Dinavahi, Noory, Gowda, Drabick, Berg, Neves, Robertson.

\section{References}

Al-Lazikani B, Banerji U, and Workman P (2012) Combinatorial drug therapy for cancer in the post-genomic era. Nat Biotechnol 30:679-692.

Cheung M, Sharma A, Madhunapantula SV, and Robertson GP (2008) Akt3 and mutant V600E B-Raf cooperate to promote early melanoma development. Cancer Res 68:3429-3439.

Chou TC (2010) Drug combination studies and their synergy quantification using the Chou-Talalay method. Cancer Res 70:440-446.

Davies BR, Greenwood H, Dudley P, Crafter C, Yu DH, Zhang J, Li J, Gao B, Ji Q, Maynard J, et al. (2012) Preclinical pharmacology of AZD5363, an inhibitor of AKT: pharmacodynamics, antitumor activity, and correlation of monotherapy activity with genetic background. Mol Cancer Ther 11:873-887.
Davies MA, Stemke-Hale K, Tellez C, Calderone TL, Deng W, Prieto VG, Lazar AJ, Gershenwald JE, and Mills GB (2008) A novel AKT3 mutation in melanoma tumours and cell lines. Br J Cancer 99:1265-1268.

Dinavahi SS, Prasanna R, Dharmarajan S, Perumal Y, and Viswanadha S (2015) A novel, potent, small molecule AKT inhibitor exhibits efficacy against lung cancer cells in vitro. Cancer Res Treat 47:913-920.

Do K, Wilsker D, Ji J, Zlott J, Freshwater T, Kinders RJ, Collins J, Chen AP, Doroshow $\mathrm{JH}$, and Kummar S (2015) Phase I study of single-agent AZD1775 (MK-1775), a Wee1 kinase inhibitor, in patients with refractory solid tumors. J Clin Oncol 33:3409-3415. Dos Santos C, Tijeras-Raballand A, Serova M, Sebbagh S, Slimane K, Faivre S, de Gramont A, and Raymond E (2015) Effects of preset sequential administrations of sunitinib and everolimus on tumour differentiation in Caki-1 renal cell carcinoma. Br J Cancer 112:86-94.

Fung AS, Yu M, Ye QJ, and Tannock IF (2013) Scheduling of paclitaxel and gefitinib to inhibit repopulation for optimal treatment of human cancer cells and xenografts that overexpress the epidermal growth factor receptor. Cancer Chemother Pharmacol 72:585-595.

Gowda R, Inamdar GS, Kuzu O, Dinavahi SS, Krzeminski J, Battu MB, Voleti SR, Amin S, and Robertson GP (2017) Identifying the structure-activity relationship of leelamine necessary for inhibiting intracellular cholesterol transport. Oncotarget 8: $28260-28277$.

Guertin AD, Li J, Liu Y, Hurd MS, Schuller AG, Long B, Hirsch HA, Feldman I, Benita Y, Toniatti C, et al. (2013) Preclinical evaluation of the WEE1 inhibitor MK-1775 as single-agent anticancer therapy. Mol Cancer Ther 12:1442-1452.

Kuzu OF, Gowda R, Sharma A, Noory MA, Dinavahi SS, Kardos G, Drabick JJ, and Robertson GP (2017a) Improving pharmacological targeting of AKT in melanoma. Cancer Lett 404:29-36.

Kuzu OF, Gowda R, Sharma A, Noory MA, Kardos G, Madhunapantula SV, Drabick JJ, and Robertson GP (2017b) Identification of WEE1 as a target to make AKT inhibition more effective in melanoma. Cancer Biol Ther DOI 10.1080/15384047.2017.1360446 [published ahead of print].

Madhunapantula SV, Sharma A, Gowda R, and Robertson GP (2013) Identification of glycogen synthase kinase $3 \alpha$ as a therapeutic target in melanoma. Pigment Cell Melanoma Res 26:886-899.

Pal SK, Reckamp K, Yu H, and Figlin RA (2010) Akt inhibitors in clinical development for the treatment of cancer. Expert Opin Investig Drugs 19:1355-1366.

Paraiso KH, Xiang Y, Rebecca VW, Abel EV, Chen YA, Munko AC, Wood E, Fedorenko IV, Sondak VK, Anderson AR, et al. (2011) PTEN loss confers BRAF inhibitor resistance to melanoma cells through the suppression of BIM expression. Cancer Res 71:2750-2760

Shao Y and Aplin AE (2010) Akt3-mediated resistance to apoptosis in B-RAF-targeted melanoma cells. Cancer Res 70:6670-6681.

Sharma A, Madhunapantula SV, Gowda R, Berg A, Neves RI, and Robertson GP (2013) Identification of aurora kinase B and Wee1-like protein kinase as downstream targets of (V600E)B-RAF in melanoma. Am J Pathol 182:1151-1162.

Song Y, Xin X, Zhai X, Xia Z, and Shen K (2015) Sequential combination of flavopiridol with Taxol synergistically suppresses human ovarian carcinoma growth. Arch Gynecol Obstet 291:143-150.

Stahl JM, Sharma A, Cheung M, Zimmerman M, Cheng JQ, Bosenberg MW, Kester M, Sandirasegarane L, and Robertson GP (2004) Deregulated Akt3 activity promotes development of malignant melanoma. Cancer Res 64:7002-7010.

Villanueva J, Vultur A, Lee JT, Somasundaram R, Fukunaga-Kalabis M, Cipolla AK, Wubbenhorst B, Xu X, Gimotty PA, Kee D, et al. (2010) Acquired resistance to $\mathrm{BRAF}$ inhibitors mediated by a RAF kinase switch in melanoma can be overcome by cotargeting MEK and IGF-1R/PI3K. Cancer Cell 18:683-695.

Visconti R, Della Monica R, and Grieco D (2016) Cell cycle checkpoint in cancer: a therapeutically targetable double-edged sword. J Exp Clin Cancer Res 35:153.

Wang D, Jiang Z, and Zhang L (2012) Concurrent and sequential administration of sunitinib malate and docetaxel in human non-small cell lung cancer cells and xenografts. Med Oncol 29:600-606.

Wang X, Zhang L, O'Neill A, Bahamon B, Alsop DC, Mier JW, Goldberg SN, Signoretti S, Atkins MB, Wood CG, et al. (2013) Cox-2 inhibition enhances the activity of sunitinib in human renal cell carcinoma xenografts. $\mathrm{Br} J$ Cancer 108: $319-326$

Watanabe N, Broome M, and Hunter T (1995) Regulation of the human WEE1Hu CDK tyrosine 15-kinase during the cell cycle. EMBO $J$ 14:1878-1891.

Wild AT, Gandhi N, Chettiar ST, Aziz K, Gajula RP, Williams RD, Kumar R, Taparra $\mathrm{K}$, Zeng J, Cades JA, et al. (2013) Concurrent versus sequential sorafenib therapy in combination with radiation for hepatocellular carcinoma. PLoS One 8:e65726.

Address correspondence to: Gavin P. Robertson, Department of Pharmacology, Pennsylvania State University College of Medicine, 500 University Drive, Hershey, PA 17033. E-mail: gpr11@psu.edu 